\title{
Added Value of SPECT/CT to Whole Body Scan Test Planar Imaging in Patients with Thyroid Cancer After Radioiodine 131 Therapy
}

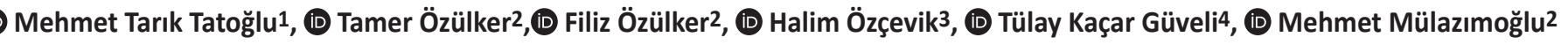 \\ 1istanbul Medeniyet University, Göztepe Training and Research Hospital, Clinic of Nuclear Medicine, Istanbul, Turkey \\ 2 University of Health Sciences Turkey, Prof. Dr. Cemil Taşçıoğlu City Hospital, Clinic of Nuclear Medicine, Istanbul, Turkey \\ 3University of Health Sciences, Kanuni Sultan Süleyman Research and Training Hospital, Clinic of Nuclear Medicine, istanbul, Turkey \\ 4 University of Health Sciences, Bakırköy Dr. Sadi Konuk Research and Training Hospital, Clinic of Nuclear Medicine, Istanbul, Turkey
}

\section{Abstract}

Objective: The aim of this retrospective study was to determine added value of single photon emission computed tomography (SPECT)/computed tomography (CT) imaging to whole body scan (WBS) planar imaging in patients with thyroid cancer after radioiodine 131 ablation therapy.

Methods: Indications for treatment of radioactive ablation therapy were postsurgical ablation, recurrent or metastatic disease with high thyroglobulin level of 50 patients with thyroid cancer were evaluated. Following 6 or 7 days after the between 50 and 300 mCi of radioiodine 131 administered orally in patients examined with WBS planar imaging, suspicion of metastasis evaluated with SPECT/CT imaging method. Imaging techniques were evaluated by nuclear medicine specialist with over 10 years experience in thyroid cancer management. The number of lesions assessed with planar images and the number of lesions assessed with SPECT/CT were recorded. The difference between the level of significance was tested using Wilcoxon Signed Rank test, SPSS 12 software package. Special cases with lesion based analysis was performed by examining the reviews.

Results: On planar images 188 foci were detected, on SPECT/CT images 196 foci were detected. Difference between the number of detected lesions was found to be a statistically significant $(p<0.05)$. SPECT/CT added diagnostic value 117 of 188 foci over planar imaging.

Conclusion: In patients with thyroid carcinoma, there was a statistically significant difference between the number of lesions detected with WBS planar imaging and the number of lesions detected with SPECT/CT.

Keywords: Radionuclide imaging, SPECT and CT, thyroid carcinoma

\section{INTRODUCTION}

The incidence of differentiated thyroid cancer (DTC) in the last three decades has increased in men and women $(1,2)$. Some studies have suggested that the use of ultrasonography and verification methods with fine needle aspiration biopsy explain this increase $(3,4)$. DTC is the most common type of cancer among endocrine system malignancies. Thyroid cancers occur at an earlier age than many adult cancer types, the median age at the time of diagnosis is 54 in men and 48 in women. The treatment method chosen in $84 \%$ of patients is total thyroidectomy, and 15\% is partial thyroidectomy. Approximately $56 \%$ of the good DTCs treated surgically receive radioactive iodine (RAI) 131 therapy for ablation of the remaining residual tissue. By suppressing the pituitary gland, suppression therapy with levothyroxine is applied to prevent the secretion of thyroidstimulating hormone (thyrotropin). In this way, recurrence rates 
are tried to be reduced. The relative 5 -year survival rate for all thyroid cancer patients is $97.3 \%$. The stage of the disease and the age of the patient at the time of diagnosis affect the survival time. The 5 -year survival rate is $99.8 \%$ in localized disease, $96.8 \%$ in regional spread, $55.4 \%$ in those with distant metastasis (5).

I-131 applied on the $6^{\text {th }}$ or $7^{\text {th }}$ day following the application of RAI ablation therapy in the DTC is included in the routine algorithm of the whole body scan (WBS) (6). Routine imaging is done as planar imaging with gamma camera. Residual tissue, lymphatic or other possible metastases can be imaged during post-treatment imaging. When imaging is done planar, there are deficiencies in terms of anatomical correlation. Due anatomical correlation and three-dimensional imaging can be done by single photon emission computed tomography (SPECT)/CT, additional lesions can be detected which may result in changes in the patient's treatment and follow-up.

The aim of this study is to show whether the contribution of SPECT/CT imaging method on planar imaging after RAI therapy in DTC patients is statistically significant and to examine possible contributions.

\section{METHODS}

This study was carried out with the approval decision of Okmeydanı Training and Research Hospital Clinical Research Ethics Committee dated 17.12.2012 and numbered 41. Consent was obtained from the patients who were scanned.

\section{Cases}

The patients who received 50-300 mCi RAl treatment in our clinic between April 2010 and October 2011 due to thyroid cancer and who underwent planar scintigraphy imaging 4 or 7 days after treatment, and from the activity involvements with suspicion of metastasis or recurrence and/or those investigated with SPECT/ CT with need of an anatomical correlation were chosen. No exclusion criteria were applied. It was observed that the patients whose dossier and epicrisis information were examined were followed up and received RAI treatments in accordance with the patient preparation protocol before RAI treatment.

\section{Imaging Technique}

1- Patient Preparation: The patients are informed about the method of scan, and the importance of their immobility throughout the study is explained.

2- Imaging: Imaging of all cases was done with Infinia double headed gamma camera or Infinia Hawkeye 4 (General Electric Medical Systems, Milwaukee, WI) double headed gamma camera. After WBS was performed, SPECT/CT examination was performed for those with suspected recurrence and metastasis, and the relevant field of view; viewing area was determined by the nuclear medicine specialist who evaluated the WBS image.

a. Planar imaging: WBS planar imaging was taken at a speed of $10 \mathrm{~cm} /$ minute, and spot images were taken in 5 minutes with Infinia double-head gamma camera or Infinia Hawkeye 4 (General Electric Medical Systems, Milwaukee, WI) double-head gamma camera.

b. SPECT/CT imaging: SPECT imaging was done with InfiniaHawkeye 4 (General Electric Medical Systems, Milwaukee, WI) device after planar imaging evaluation. With wide field of view, high energy, high resolution, parallel hole collimator, 60 images were taken in total 360 degrees with zoom value at 1 , peak energy level $364 \mathrm{KeV}$, window range 10\%, each image 30 seconds, 128x128 matrix, 6 degrees step and shoot mode. CT imaging was performed with Infinia-Hawkeye 4 (General Electric Medical Systems, Milwaukee, WI) device without giving oral or intravenous contrast agent immediately after SPECT imaging. In the system containing low dose CT (4 slice), the settings of the $\mathrm{X}$-ray tube were determined at $140 \mathrm{kV}$ and $2.5 \mathrm{~mA}$, in the mode of scan type being helical, with a wide viewing angle in the pitch of 1.9, 512x512 matrix in half rotation. Standard filter is applied to the images obtained. The scan time lasted about 4.5 minutes for $40 \mathrm{~cm}$ in helical shooting.

\section{Evaluation Criteria}

Planar and SPECT/CT images were evaluated by a nuclear medicine specialist with more than 10 years of experience in thyroid cancer imaging. Apart from nuclear medicine scintigraphic images, clinical information, laboratory values and available radiological images were also taken into consideration during the evaluation.

During the evaluation of scintigraphic findings, one or more activity areas with more involvement than background activity were positively evaluated. Nasal activity was considered normal. Symmetrical salivary gland activity, gastrointestinal activities, bladder activity were evaluated as normal. Diffuse activity involvement in the liver was considered normal in people with functional thyroid tissue. The activity involvement areas in other areas were recorded as abnormal.

SPECT images were performed on Xeleris (General Electric Medical Systems, Milwaukee, WI) workstation. Qrecon Quant filter was used as reconstruction downhill filter. After filtering with the Hann prereconstruction filter (frequency value 0.9), it 
is reconstructed with OSEM iterative image refresh technique, while the subset value was 10 and refresh value was 2 . Filtered with Hann 3D post reconstruction filter (frequency value 0.9). Fusion images were obtained by combining the SPECT images with attenuation correction by computer and $\mathrm{CT}$ images. It was evaluated whether SPECT/CT images are synchronized with regions with RAI involvement in transverse, coronal, sagittal sections and 3D images.

Lesion numbers were recorded in both imaging methods for statistical analysis. For each lesion, it was classified as malignant or benign by making anatomical correlation with SPECT/CT and the contribution rate of SPECT/CT was recorded in the lesionbased analysis.

\section{Statistical Analysis}

SPSS 12.0 software package was used for statistical analysis. In order to determine the appropriate test to be used in the evaluation of the difference in the number of lesions between planar imaging and SPECT/CT imaging, it was first examined whether the variables were normally distributed or not. The Wilcoxon Signed Rank test was used to analyze the difference in the number of lesions, since the normal distribution assumption did not occur. Hypotheses for testing were established as follows.

$\mathrm{H}_{0}$ : There is no statistical difference between the number of lesions determined by planar imaging and the number of lesions determined by SPECT/CT imaging.

$\mathrm{H}_{1}$ : There is a statistical difference between the number of lesions determined by planar imaging and the number of lesions determined by SPECT/CT imaging.

$95 \%$ significance level $(p<0.05)$ was used in the tests.

Lesion-based analysis was performed. Differences between the planar imaging method and SPECT/CT were recorded. The contribution value of SPECT/CT was calculated as a percentage.

\section{RESULTS}

The cases were between the ages of 18-80. The average age was calculated as $47.60 \pm 16.05$ (Table 1). Of the 50 patients, 42 (84\%) were female and 8 (16\%) were male (Figure 1).

A total of 188 lesions were detected on planar images and 196 lesions on SPECT/CT images (Table 2).

\begin{tabular}{|l|l|l|l|l|l|}
\hline \multicolumn{6}{|l|}{ Table 1. Distribution of cases by age } \\
\hline & $\mathbf{n}$ & Minimum & Maximum & Average & $\begin{array}{l}\text { Standard } \\
\text { deviation }\end{array}$ \\
\hline Age & 50 & 18 & 80 & 47.60 & 16,05475 \\
\hline
\end{tabular}

The dose of RAI applied to the cases is in the range of 50-300 $\mathrm{mCi}$. The average RAI dose was calculated as $131.40 \mathrm{mCi}$.

There was a statistically significant difference in terms of the number of lesions determined by planar imaging and SPECT/CT imaging ( $p>0.05)$. The test results are shown below (Table 3 ).

Lesion-based analysis; contribution after evaluation of lesions considered suspicious in planar imaging with SPECT/CT; a total of 188 lesions were detected in planar imaging, and 196 lesions in SPECT/CT imaging. When 117 lesions considered suspicious in planar imaging were evaluated by SPECT/CT, 44 were defined as benign and 73 as malignant lesions.

Anatomic correlation was performed for all other lesions in the SPECT/CT imaging area (Table 4).

\section{DISCUSSION}

Unlike other imaging modalities, there are unique methods of defining physiological activities and processes using nuclear medicine techniques. Most of the time, knowing the physiological activity involvement helps in the interpretation of the examination. When an unusual involvement is detected in imaging, it is important to determine the exact localization of the lesion for the planning of treatment. Determination of the exact localization has become possible today with the SPECT/CT imaging method (7). By combining anatomical and molecular imaging methods with devices such as SPECT/CT and $\mathrm{PET} / \mathrm{CT}$, more precise comments can be made regarding better attenuation correction, better anatomic correlation and examinations (7).

In thyroid cancer patients, WBS performed on the $5^{\text {th }}-8^{\text {th }}$ days after RAl ablation therapy is used as a standard imaging method. Important information about the condition of the disease is

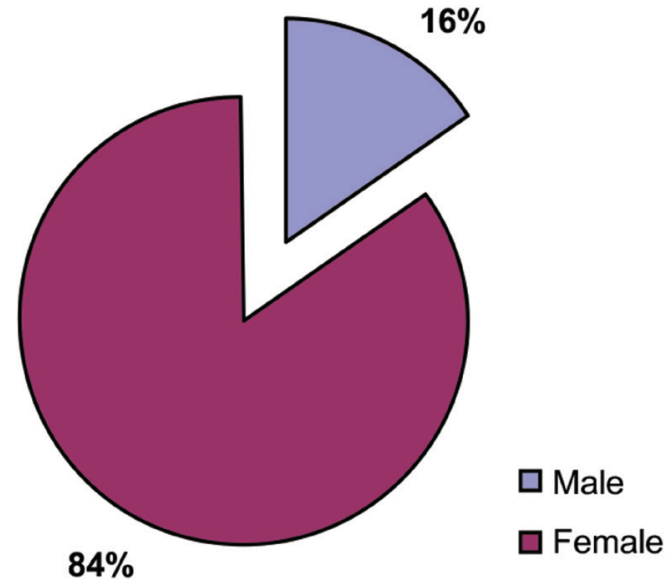

Figure 1. Distribution of cases by gender 


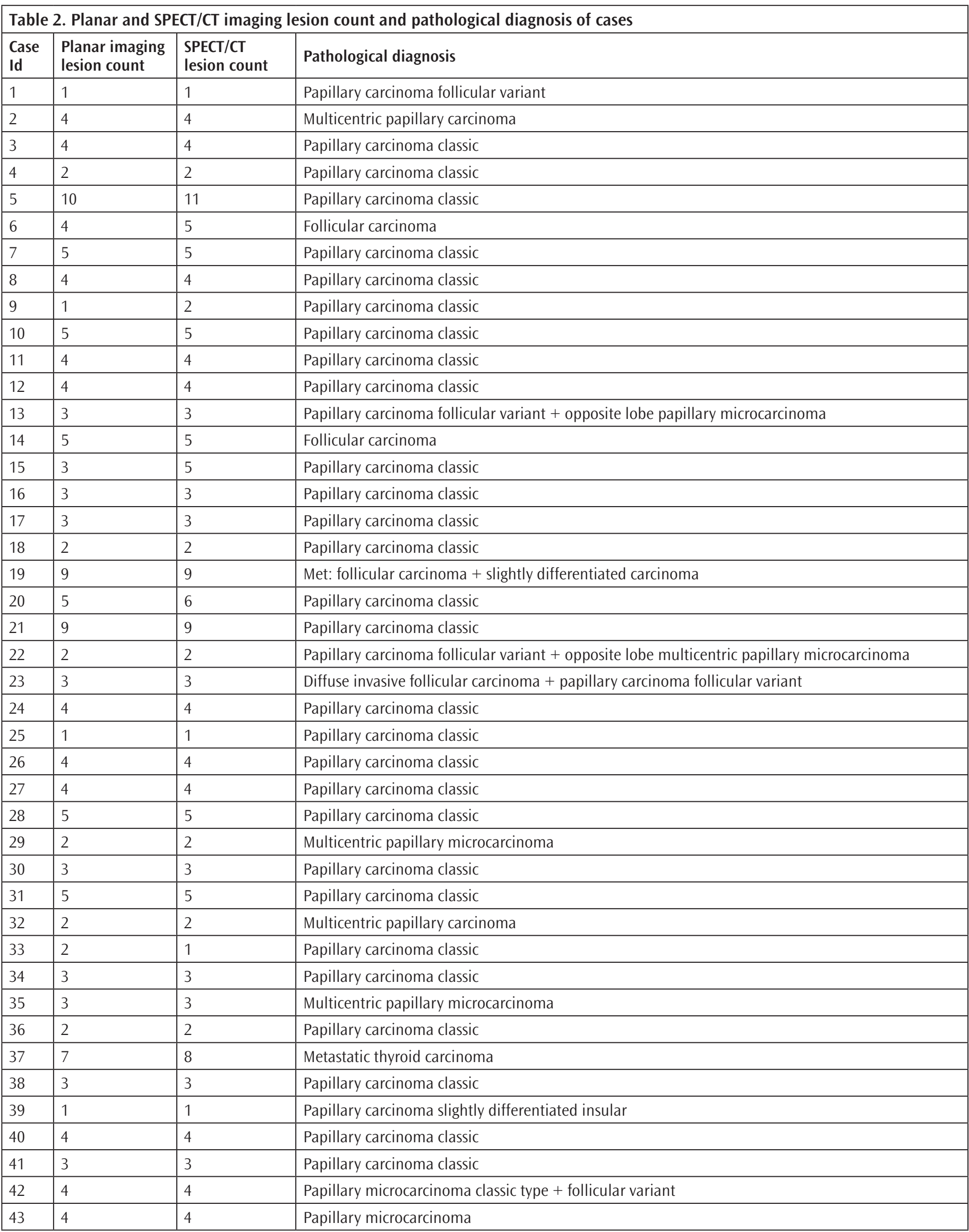




\begin{tabular}{|l|l|l|l|}
\hline \multicolumn{4}{|l|}{ Table 2. Continued } \\
\hline $\begin{array}{l}\text { Case } \\
\text { Id }\end{array}$ & $\begin{array}{l}\text { Planar imaging } \\
\text { lesion count }\end{array}$ & $\begin{array}{l}\text { SPECT/CT } \\
\text { lesion count }\end{array}$ & Pathological diagnosis \\
\hline 44 & 2 & 2 & Minimally invasive follicular carcinoma \\
\hline 45 & 3 & 3 & Papillary carcinoma classic \\
\hline 46 & 5 & 5 & Mixed type of papillary carcinoma \\
\hline 47 & 9 & 11 & Papillary carcinoma classic \\
\hline 48 & 2 & 2 & Papillary carcinoma classic \\
\hline 49 & 3 & 3 & Papillary carcinoma classic \\
\hline 50 & 3 & 3 & Papillary carcinoma follicular variant \\
\hline Total & 188 & 196 & \\
\hline SPECT: Single-photon emission computed tomography, CT: Computed tomography \\
\hline
\end{tabular}

\begin{tabular}{|c|c|c|c|c|}
\hline \multicolumn{5}{|l|}{ Ranks } \\
\hline & & n & $\begin{array}{l}\text { Mean } \\
\text { Rank }\end{array}$ & $\begin{array}{l}\text { Sum of } \\
\text { Ranks }\end{array}$ \\
\hline \multirow[t]{4}{*}{ SPECT-CT - planar } & Negative ranks & 1 (a) & 3.50 & 3.50 \\
\hline & Positive ranks & 7 (b) & 4.64 & 32.50 \\
\hline & Ties & $42(c)$ & & \\
\hline & Total & 50 & & \\
\hline \multicolumn{5}{|l|}{ Test statistics } \\
\hline & \multicolumn{4}{|l|}{ SPECT-CT - planar } \\
\hline Z & \multicolumn{4}{|l|}{$-2.126(\mathrm{a})$} \\
\hline $\begin{array}{l}\text { Asymp. Sig. } \\
\text { (2-tailed) }\end{array}$ & \multicolumn{4}{|l|}{0.033} \\
\hline \multicolumn{5}{|c|}{$\begin{array}{l}\text { a SPECT-CT < planar, b SPECT-CT > planar, c SPECT-CT=planar } \\
\text { a: Based on negative ranks, b: Wilcoxon signed ranks test }\end{array}$} \\
\hline
\end{tabular}

\begin{tabular}{|l|l|l|l|}
\hline \multicolumn{2}{|l|}{ Table 4. Lesion-based analysis } \\
\hline & $\begin{array}{l}\text { Lesion } \\
\text { count }\end{array}$ & $\begin{array}{l}\text { Impact of SPECT/ } \\
\text { CT }\end{array}$ & $\begin{array}{l}\text { Contribution } \\
\text { percentage }\end{array}$ \\
\hline $\begin{array}{l}\text { Total lesion - planar/ } \\
\text { SPECT-CT }\end{array}$ & $188 / 196$ & $\begin{array}{l}\text { Increase in lesion } \\
\text { detection }\end{array}$ & $4.3 \%$ \\
\hline $\begin{array}{l}\text { Diagnostic } \\
\text { contribution of } \\
\text { SPECT/CT }\end{array}$ & $117 / 188$ & $\begin{array}{l}\text { Increased } \\
\text { determination in } \\
\text { localization and } \\
\text { characterization } \\
\text { (benign/ } \\
\text { malignant) }\end{array}$ & $62.2 \%$ \\
\hline $\begin{array}{l}\text { Anatomical } \\
\text { Correlation }\end{array}$ & $71 / 188$ & $\begin{array}{l}\text { Increase in } \\
\text { localization } \\
\text { determination }\end{array}$ \\
\hline \multicolumn{3}{|l|}{ SPECT: Single-photon emission computed tomography, CT: Computed tomography } \\
\hline
\end{tabular}

obtained after treatment with WBS. Although RAI involvement is specific for thyroid tissue, many false positive conditions such as contaminations, physiological excretions can occur in planar scintigraphy, and a definite anatomical correlation is needed to distinguish between a physiological condition and a malignant lesion. By determining the exact anatomical localization of RAI involvement with SPECT/CT, diagnostic accuracy is increased and a positive contribution is made to the management of patients' treatment (8-10). Before the clinical use of the SPECT/CT device became widespread, it was attempted to examine SPECT and CT by using computer fusions after performing on separate devices, and to use external markers outside the body, and publications have been published on the usefulness of these $(11,12)$.

In this study, the cases with suspicious involvements during WBS planar imaging performed after RAI ablation therapy in thyroid cancer patients were evaluated for those who were identified and/or anatomically correlated with SPECT/CT. It is aimed to examine the contributions of SPECT/CT to planar imaging. For this purpose, whether there is a statistical difference between the number of lesions detected in planar imaging and the number of lesions detected in SPECT/CT was tested with the SPSS 12.0 package program Wilcoxon Signed Rank test and the result was found to be statistically significant. The total number of lesions detected in planar imaging was 188, the number of lesions detected in SPECT/CT was 196, and the rate of increase was calculated as $4.3 \%$. In addition, the contribution of SPECT/CT was evaluated by performing lesion-based analysis. According to the result of the lesion-based analysis; it was found that SPECT/ CT was useful in defining RAl involvement in 117 (62.2\%) of 188 lesions detected in planar imaging. It has been observed that it also contributed to the anatomical correlation of all remaining foci of involvement (27.8\%).

In a study conducted by Ruf et al. (13), which included 24 thyroid cancer patients who could not be concluded with full-body-scan planar imaging after RAl ablation therapy $(3.7 \mathrm{GBq}=100 \mathrm{mCi}$ ); by comparing SPECT and SPECT/CT imaging methods, it was examined whether fusion imaging contributed. As a result of the study, in focus-based examination; SPECT/CT provided important clinical information by providing comment changes in 15 (38\%) 
out of 39 focal points. In patient-based examination; these findings were found to be significant in $6(25 \%)$ of 24 patients, and SPECT/CT fusion was suggested to rule out artifacts. Compared to this study, it is seen that similar results are obtained. It was thought that the variance in contribution percentage of SPECT/ CT may be related to the selection of patients.

In the study conducted by Schmidt et al. (14) to evaluate the contribution of SPECT/CT to nodal staging in 57 thyroid cancer patients after RAI ablation therapy; the cervical region was evaluated with planar imaging and SPECT/CT. SPECT/CT led to a revision in the original diagnosis of 28 of the 143 RAI involvement foci detected in planar imaging. In particular, it classified 6 of the 11 focal points considered as lymph node metastasis in planar imaging and 11 of the 15 unidentified focuses as benign, and identified 11 focal points as lymph node metastasis which were previously defined as thyroid balance or ambiguous in planar imaging. SPECT/CT contributed to nodal staging in 20 (35\%) of 57 patients, and changed the nodal staging from N0 to $\mathrm{N} 1$ in 2 of 20 patients and from $\mathrm{N}$ ambiguous (Nx) to $\mathrm{N} 1$ in 6 of 30 patients. According to these results, it changed the risk layer in 14 of 57 patients according to International Union Against Cancer. Although nodal staging was not taken into consideration in this study, a full comparison could not be made, but 117 of 188 focal points detected in planar imaging were identified as suspicious, and 44 of these foci were classified as benign and 73 were malignant with SPECT/CT, which suggests that these two methods provide information in the same direction.

In the study conducted by Aide et al. (15), 55 thyroid cancer patients undergoing RAI treatment were evaluated and it was shown that cases identified as ambiguous by planar imaging decreased from $29 \%$ to $7 \%$ with SPECT/CT. In addition, compared to planar scintigraphy, SPECT/CT has been shown to be superior in predicting treatment failure. In this study, there was no lesion that SPECT/CT could not identify among the involvement detected uncertain in planar imaging.

In a dicentric study of Mustafa et al. (16), 151 cases of T1 stage papillary carcinoma, 96 of which were papillary microcarcinoma were evaluated; cervical lymph node dissection was performed in 69 patients and SPECT/CT based nodal staging was performed in the remaining 82 patients. The incidence of lymph node metastasis was $26 \%$ in the whole group and $22 \%$ in the papillary microcarcinoma group. It was determined that SPECT/CT provides more accurate information in $24.5 \%$ of patients compared to planar scintigraphy. In this study, no comment was made on the diagnostic accuracy of SPECT/CT in nodal staging due to the absence of histopathological verification.
In the study conducted by Ciappuccini et al. (17) on 170 DTC patients; after RAI ablation therapy, the effects of other prognostic factors such as age, gender, stimulated triglyceride (Tg) level, tumor, lymph node metastasis, macroscopic lymph node involvement on disease-free survival with the neck and thorax SPECT/CT examination were compared. WBS showing positive RAl involvement only and A Tg negative, Tg level at least $58 \mathrm{ng} / \mathrm{dL}$ were found to be associated with an increased risk of recurrence or persistent disease.

Chen et al. (18) performed 37 SPECT/CT studies where 23 patients with locally advanced or metastatic thyroid carcinoma failed to achieve any results with planar imaging after RAI ablation therapy; they found that it contributed to the diagnosis in 17 of 23 patients (74\%). They reported that treatment strategy changed in 8 (35\%) of 23 patients with SPECT/CT imaging. They reported that the diagnostic accuracy of fusion SPECT/CT imaging was greater than planar scintigraphy, reduced trap images, and caused a change in treatment strategy.

It was observed that the findings detected in many studies and the findings found in this study were compatible and supportive. In this study, it was seen that SPECT/CT is a contributing imaging method for characterizing suspicious involvements in planar imaging and making anatomical correlations.

In the clinic where the study was performed, the indication for performing SPECT/CT examination in patients included in the study was suspicious involvement in WBS and the need for anatomical correlation (Figure 2-4). After RAI ablation therapy of thyroid cancer patients, WBS testing continues to be performed with planar imaging as a routine application in the patient group. In other words, SPECT/CT imaging is not routinely applied to all patients. The reason why it is not routinely applied in all patient groups; although the CT component of the SPECT/CT device produces lower dose radioactivity than conventional CT imaging devices, it is to keep the radiation dosimetry low. It was mentioned earlier in this section that there were publications that showed that SPECT/CT detected lesions that could not be detected in the planar image and lesions in even WBS negative cases. In the clinic where this study was performed, WBS negative patients could not be included in the study because SPECT/CT was not performed. It may be thought that the patient group of the cases with suspicious findings in WBS, which caused the narrowing of the patient group in the study. It was thought that studies should be conducted in multi-centered, wider patient groups in order to comment on whether SPECT/CT should be applied to all cases as a routine practice. 


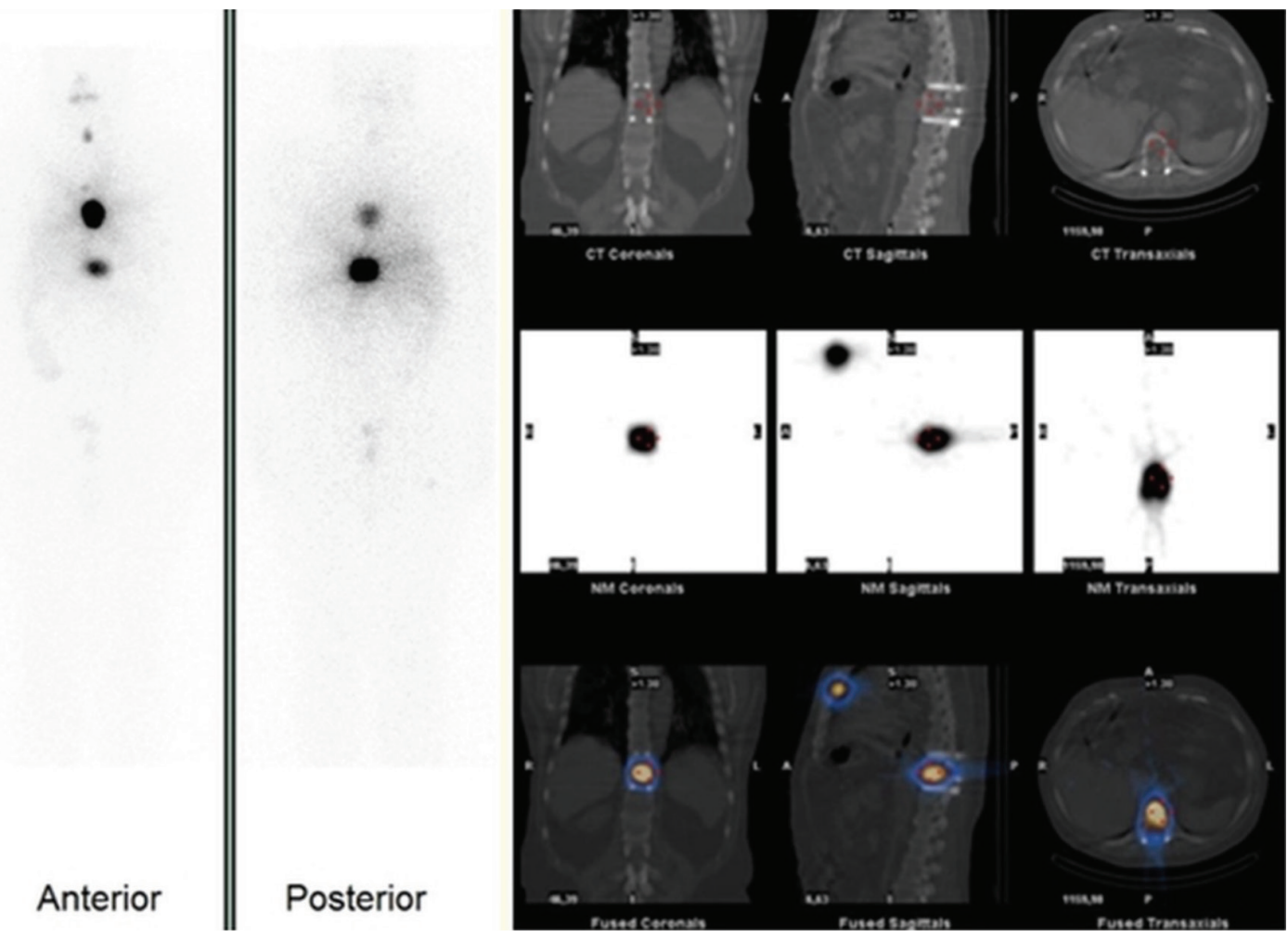

Figure 2. A 56-year-old male who had papillary carcinoma metastasis as a result of the pathology of the mass excision from the dorsal vertebra 16 months ago, underwent bilateral total thyroidectomy 14 months ago. The patient, whose pathology result was reported as follicular variant of papillary carcinoma, was administered 150 mCi RAI treatment. Local recurrence and lung metastasis were detected in I-131 WBS during follow-up. In the WBS performed on the $7^{\text {th }}$ day of the patient who received $200 \mathrm{mCi}$ RAI treatment, mild RAI involvement in the thyroid lodge, severe RAI involvement in the midline in the mediastinum and mild RAI involvement in the right of the midline and more severe RAI involvement in the posterior position were observed in the dorsal area. In the planar image with SPECT/CT, it was observed that RAI involvement observed in the middle part of the mediastinum was in the sternum, and the involvement in the dorsal area was in the D10-11 vertebrae. In the CT component of SPECT/CT, the appearance of the screws placed in the operation through the dorsal vertebra is observed. With SPECT/CT, the location of RAI involvement was precisely determined and anatomical correlation was made. Lesions were reported as metastasis

RAI: Radioactive iodine, WBS: Whole body scan, SPECT: Single-photon emission computed tomography CT: Computed tomography

In this study, 188 RAI involvement focuses were detected in WBS planar imaging, and $196 \mathrm{RAI}$ involvement focuses were detected with SPECT/CT, and it was found that there was a statistically significant difference. SPECT/CT provided additional clinical information or helped lesion characterization in 117 of 188 RAI involvements observed on WBS planar imaging. The contribution of found foci on staging has not been studied separately, the first reason for this was there were patients who were at a high stage in the patient group and who underwent RAI due to high Tg at follow-up, and second reason was the idea that it would be more meaningful to do it in a larger patient series. It has been observed that the CT component of SPECT/CT contributes by changing the treatment course in some cases. For example, in one case, the pulmonary consolidation area was monitored, and the focus detected in favor of metastasis in the consolidation area, and it was seen that it could make changes in the course of treatment.

\section{CONCLUSION}

It is thought that conducting such researches, which will enable the role of SPECT/CT to be better determined in the process of thyroid cancer follow-up, may contribute to better follow-up of doctors. According to this study, SPECT/CT is a recommended imaging method for lesions in which planar imaging is suspected or requires anatomical correlation after RAI ablation therapy of thyroid cancer.

\section{Acknowledgement}

This article was compiled from the medical speciality thesis titled "Added value of SPECT/CT to whole body scan test planar imaging in patient with thyroid cancer after radioiodine 131 therapy", presented by Mehmet Tarık Tatoğlu at Nuclear Medicine Clinic of Okmeydanı Training and Research Hospital. 


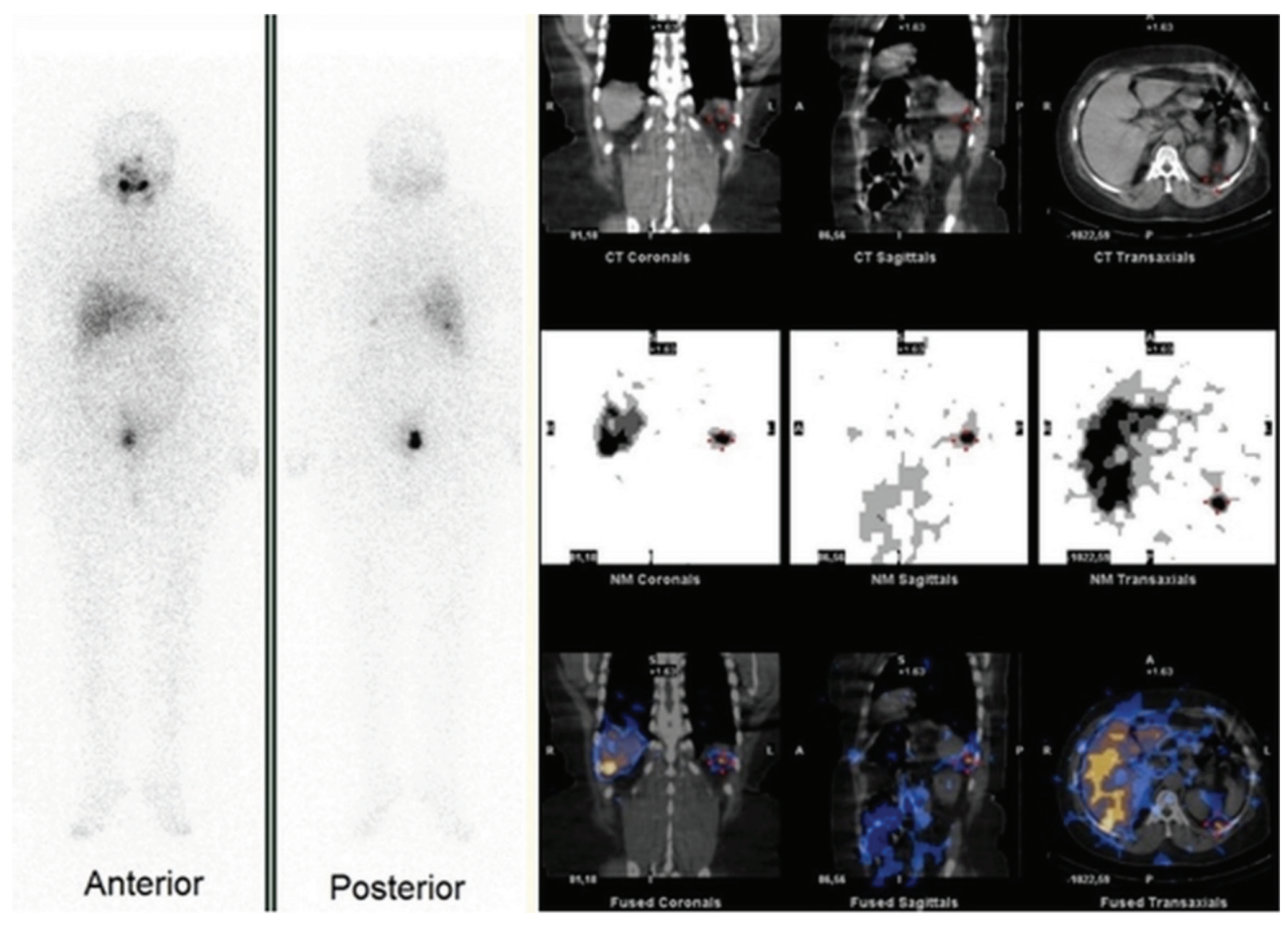

Figure 3. A 55-year-old female who underwent bilateral total thyroidectomy and received $100 \mathrm{mCi}$ RAl ablation therapy due to the pathology result of multicentric papillary carcinoma, SPECT/CT imaging was performed due to the detection of suspicious foci on the right and left sides of the abdomen in the WBS on the $7^{\text {th }}$ day of the treatment. In SPECT/CT images, one intense RAI involvement was observed in the $6^{\text {th }}$ segment of the liver and adjacent to the ribs in the inferoposterior of the spleen. In planar imaging with SPECT/CT, the RAI involvements observed as suspicious in the abdomen were anatomically correlated and metastasis was reported

RAI: Radioactive iodine, WBS: Whole body scan, SPECT: Single-photon emission computed tomography CT: Computed tomography

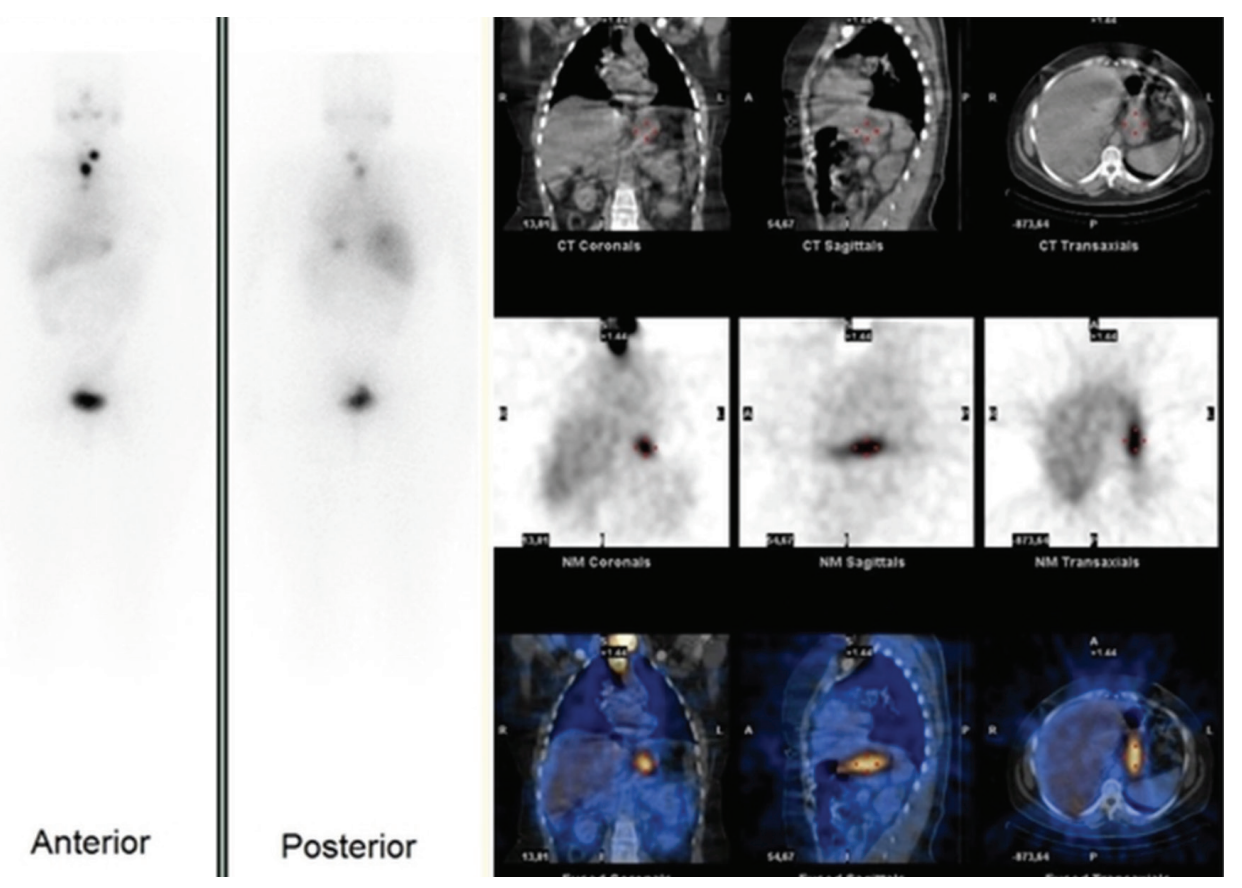

Figure 4. RAI treatment due to the pathology result of papillary carcinoma with capsular invasion and vascular invasion, SPECT/CT imaging was performed due to suspicious RAI involvement in the upper mediastinum compatible with the residual tissue in the thyroid lodge and in the left midline in the abdomen in the WBS performed on the $7^{\text {th }}$ day of the treatment. SPECT/CT images showed an involvement consistent with metastatic LAP in the upper mediastinum, while benign activity involvement consistent with the stomach was observed in the left midline of the abdomen. With $\mathrm{SPECT} / \mathrm{CT}$, in this case one suspect involvement was reported as malignant and one suspicious involvement was reported as benign RAI: Radioactive iodine, WBS: Whole body scan, SPECT: Single photon emission computed tomography CT: Computed tomography, LAP: Lymphadenopathy 


\section{Ethics}

Ethics Committee Approval: Okmeydanı Training and Research Hospital Clinical Research Ethics Committee dated 17.12.2012 and numbered 41.

Informed Consent: Form was obtained from the patients.

Peer-review: Externally peer-reviewed.

\section{Authorship Contributions}

Surgical and Medical Practices: M.T.T., T.Ö., F.Ö., M.M., Concept: M.T.T., T.Ö., M.M., Design: M.T.T., T.Ö., M.M., Data Collection or Processing: M.T.T., H.Ö., Analysis or Interpretation: M.T.T., T.Ö., F.Ö., M.M., H.Ö., T.K.G., Literature Search: M.T.T., Writing: M.T.T.

Conflict of Interest: No conflict of interest was declared by the authors.

Financial Disclosure: The authors declared that this study received no financial support.

\section{REFERENCES}

1. Chen AY, Jemal A, Ward EM. Increasing incidence of differantiated thyroid cancer in the United States, 1988-2005. Cancer 2009;115:38017.

2. Siegel R, DeSantis C, Virgo K, Stein K, Mariotto A, Smith T, et al. Cancer treatment and survivorship statistics, 2012. CA Cancer J Clin 2012;62:22041.

3. Davies L, Ouellette M, Hunter M, Welch HG. The increasing incidence of small thyroid cancers: where are the cases coming from? Laryngoscope 2010;120:2446-51.

4. Davies L, Welch HG. Increasing incidence of thyroid cancer in the United States, 1973-2002. JAMA 2006; 295:2164-7.

5. Siegel R, DeSantis C, Virgo K, Stein K, Mariotto A, Smith T, et al. Cancer Statistics, 2012. CA Cancer J Clin 2012;62:220-41.

6. American Thyroid Association (ATA) Guidelines Taskforce on Thyroid Nodules and Differentiated Thyroid Cancer, Cooper DS, Doherty GM, Haugen BR, Kloos RT, Lee SL, et al. Revised American Thyroid Association management guidelines for patients with thyroid nodules and differentiated thyroid cancer. Thyroid 2009;19:1167-214.
7. Patton JA, Turkington TG. SPECT/CT physical principles and attenuation correction. J Nucl Med Technol 2008;36:1-10.

8. Roarke MC, Nguyen BD, Pockaj BA. Applications of SPECT/CT in nuclear radiology. AJR Am J Roentgenol 2008;191:135-50.

9. Delbeke D, Schöder H, Martin WH, Wahl RL. Hybrid imaging (SPECT/ CT and PET/CT): improving therapeutic decisions. Semin Nucl Med 2009;39:308-40.

10. Seo Y, Mari C, Hasegawa BH. Technological Development and Advances in SPECT/CT. Semin Nucl Med 2008;38:177-98.

11. Pérault C, Schvartz C, Wampach H, Liehn JC, Delisle MJ. Thoracic and abdominal SPECT-CT image fusion without external markers in endocrine carcinomas. The Group of Thyroid Tumoral Pathology of Champagne-Ardenne. J Nucl Med 1997;38:1234-42.

12. Yamamoto $Y$, Nishiyama $Y$, Monden T, Matsumura $Y$, Satoh K, Ohkawa M. Clinical usefulness of fusion of 131I SPECT and CT images in patients with differentiated thyroid carcinoma. J Nucl Med 2003;44:1905-10.

13. Ruf J, Lehmkuhl L, Bertram H, Sandrock D, Amthauer H, Humplik B, et al. Impact of SPECT and integrated low-dose CT after radioiodine therapy on the management of patients with thyroid carcinoma. Nucl Med Commun 2004;25:1177-82.

14. Schmidt D, Szikszai A, Linke R, Bautz W, Kuwert T. Impact of 131I SPECT/ spiral CT on nodal staging of differentiated thyroid carcinoma at the first radioablation. J Nucl Med 2009;50:18-23.

15. Aide N, Heutte N, Rame JP, Rousseau E, Loiseau C, Amar MH, et al. Clinical relevance of single-photon emission computed tomography/ computed tomography of the neck and thorax in postablation (131)I scintigraphy for thyroid cancer. J Clin Endocrinol Metab 2009; 94:207584.

16. Mustafa M, Kuwert T, Weber K, Knesewitsch P, Negele T, Haug A, et al. Regional lymph node involvement in $\mathrm{T} 1$ papillary thyroid carcinoma: a bicentric prospective SPECT/CT study. Eur J Nucl Med Mol Imaging 2010;37:1462-6.

17. Ciappuccini R, Heutte N, Trzepla G, RAme JP, Vaur D, Aide N, et al. Postablation (131) I scintigraphy with neck and thorax SPECT-CT and stimulated serum thyroglobulin level predict the outcome of patients with differentiated thyroid cancer. Eur J Endocrinol 2011;164:961-9.

18. Chen L, Luo Q, Shen Y, Yu Y, Yuan Z, Lu H, et al. Incremental value of 131 I SPECT/CT in the management of patients with differentiated thyroid carcinoma. J Nucl Med 2008;49:1952-7. 